\title{
Convergence of greedy approximation II. The trigonometric system
}

by

\author{
S. V. Konyagin (Moscow) and V. N. Temlyakov (Columbia, SC)
}

\begin{abstract}
We study the following nonlinear method of approximation by trigonometric polynomials. For a periodic function $f$ we take as an approximant a trigonometric polynomial of the form $G_{m}(f):=\sum_{k \in \Lambda} \widehat{f}(k) e^{i(k, x)}$, where $\Lambda \subset \mathbb{Z}^{d}$ is a set of cardinality $m$ containing the indices of the $m$ largest (in absolute value) Fourier coefficients $\widehat{f}(k)$ of the function $f$. Note that $G_{m}(f)$ gives the best $m$-term approximant in the $L_{2}$-norm, and therefore, for each $f \in L_{2},\left\|f-G_{m}(f)\right\|_{2} \rightarrow 0$ as $m \rightarrow \infty$. It is known from previous results that in the case of $p \neq 2$ the condition $f \in L_{p}$ does not guarantee the convergence $\left\|f-G_{m}(f)\right\|_{p} \rightarrow 0$ as $m \rightarrow \infty$. We study the following question. What conditions (in addition to $\left.f \in L_{p}\right)$ provide the convergence $\left\|f-G_{m}(f)\right\|_{p} \rightarrow 0$ as $m \rightarrow \infty$ ? In the case $2<p \leq \infty$ we find necessary and sufficient conditions on a decreasing sequence $\left\{A_{n}\right\}_{n=1}^{\infty}$ to guarantee the $L_{p}$-convergence of $\left\{G_{m}(f)\right\}$ for all $f \in L_{p}$ satisfying $a_{n}(f) \leq A_{n}$, where $\left\{a_{n}(f)\right\}$ is the decreasing rearrangement of the absolute values of the Fourier coefficients of $f$.
\end{abstract}

1. Introduction. We study the following natural nonlinear method of summation of trigonometric Fourier series. Consider a periodic function $f \in L_{p}\left(\mathbb{T}^{d}\right), 1 \leq p \leq \infty\left(L_{\infty}\left(\mathbb{T}^{d}\right)=C\left(\mathbb{T}^{d}\right)\right)$, defined on the $d$-dimensional torus $\mathbb{T}^{d}$. Let $m \in \mathbb{N}$ and $t \in(0,1]$ be given, and let $\Lambda_{m}$ be a set of $k \in \mathbb{Z}^{d}$ with the properties:

$$
\min _{k \in \Lambda_{m}}|\widehat{f}(k)| \geq t \max _{k \notin \Lambda_{m}}|\widehat{f}(k)|, \quad\left|\Lambda_{m}\right|=m,
$$

where

$$
\widehat{f}(k):=(2 \pi)^{-d} \int_{\mathbb{T}^{d}} f(x) e^{-i(k, x)} d x
$$

is the $k$ th Fourier coefficient of $f$. We define

$$
G_{m}^{t}(f):=G_{m}^{t}(f, \mathcal{T}):=S_{\Lambda_{m}}(f):=\sum_{k \in \Lambda_{m}} \widehat{f}(k) e^{i(k, x)}
$$

2000 Mathematics Subject Classification: 42B08, 42B35.

This research was supported by the National Science Foundation Grant DMS 0200187 and by ONR Grant N00014-96-1-1003. 
and call it an $m$ th weak greedy approximant of $f$ with regard to the trigonometric system $\mathcal{T}:=\left\{e^{i(k, x)}\right\}_{k \in \mathbb{Z}^{d}}$. We write $G_{m}(f)=G_{m}^{1}(f)$ and call it an $m$ th greedy approximant. Clearly, an $m$ th weak greedy approximant and even an $m$ th greedy approximant may not be unique. In this paper we do not impose any extra restrictions on $\Lambda_{m}$ in addition to (1.1). Thus the theorems formulated below hold for any choice of $\Lambda_{m}$ satisfying (1.1) or in other words for any realization $G_{m}^{t}(f)$ of the weak greedy approximation.

There has recently been much interest in approximation of functions by $m$-term approximants with regard to a basis (or a minimal system; see surveys $[\mathrm{D}]$ and $[\mathrm{T} 2])$. We will discuss in detail only results concerning the trigonometric system. T. W. Körner, answering a question raised by Carleson and Coifman, constructed in [K1] a function from $L_{2}(\mathbb{T})$ and then in $[\mathrm{K} 2]$ a continuous function such that $\left\{G_{m}(f, \mathcal{T})\right\}$ diverges almost everywhere. It has been proved in [T1] for $p \neq 2$ and in [CF] for $p<2$ that there exists $f \in L_{p}(\mathbb{T})$ such that $\left\{G_{m}(f, \mathcal{T})\right\}$ does not converge in $L_{p}$. It was remarked in [T2] that the method from [T1] gives a little more: 1$)$ There exists a continuous function $f$ such that $\left\{G_{m}(f, \mathcal{T})\right\}$ does not converge in $L_{p}(\mathbb{T})$ for all $\left.p>2 ; 2\right)$ There exists a function $f$ that belongs to all $L_{p}(\mathbb{T})$, $p<2$, such that $\left\{G_{m}(f, \mathcal{T})\right\}$ does not converge in measure. Thus the above negative results show that the condition $f \in L_{p}\left(\mathbb{T}^{d}\right), p \neq 2$, does not guarantee the convergence of $\left\{G_{m}(f, \mathcal{T})\right\}$ in the $L_{p}$-norm. The main goal of this paper is to find an additional condition on $f$ (besides $f \in L_{p}$ ) to guarantee that $\left\|f-G_{m}(f, \mathcal{T})\right\|_{p} \rightarrow 0$ as $m \rightarrow \infty$. In Section 2 we prove the following theorem.

Theorem 1. Let $f \in L_{p}\left(\mathbb{T}^{d}\right), 2<p \leq \infty$, and let $q>p^{\prime}:=p /(p-1)$. Assume that

$$
\sum_{|k|>n}|\widehat{f}(k)|^{q}=o\left(n^{d\left(1-q / p^{\prime}\right)}\right)
$$

where $|k|:=\max _{1 \leq j \leq d}\left|k_{j}\right|$. Then

$$
\lim _{m \rightarrow \infty}\left\|f-G_{m}^{t}(f, \mathcal{T})\right\|_{p}=0 .
$$

For $f \in L_{1}\left(\mathbb{T}^{d}\right)$ let $\{\widehat{f}(k(l))\}_{l=1}^{\infty}$ denote the decreasing rearrangement of $\{\widehat{f}(k)\}_{k \in \mathbb{Z}^{d}}$, i.e.

$$
|\widehat{f}(k(1))| \geq|\widehat{f}(k(2))| \geq \ldots
$$

Set $a_{n}(f):=|\widehat{f}(k(n))|$. In Section 3 we prove the following theorem.

Theorem 2. Let $2<p<\infty$ and let $\left\{A_{n}\right\}_{n=1}^{\infty}$ be a decreasing sequence satisfying

$$
A_{n}=o\left(n^{1 / p-1}\right) \quad \text { as } n \rightarrow \infty .
$$


Then for any $f \in L_{p}\left(\mathbb{T}^{d}\right)$ with $a_{n}(f) \leq A_{n}$ for $n=1,2, \ldots$, we have

$$
\lim _{m \rightarrow \infty}\left\|f-G_{m}^{t}(f, \mathcal{T})\right\|_{p}=0 .
$$

We also prove in Section 3 that for any decreasing sequence $\left\{A_{n}\right\}$ satisfying

$$
\limsup _{n \rightarrow \infty} A_{n} n^{1-1 / p}>0
$$

there exists a function $f \in L_{p}$ with $a_{n}(f) \leq A_{n}$ for $n=1,2, \ldots$ and with the sequence $\left\{G_{m}(f)\right\}$ of greedy approximants divergent in $L_{p}$.

In Section 4 we prove a necessary and sufficient condition on the majorant $\left\{A_{n}\right\}$ to guarantee (under the assumption that $f$ is continuous) the uniform convergence of the greedy approximants to the function $f$.

THEOREM 3. Let $\left\{A_{n}\right\}_{n=1}^{\infty}$ be a decreasing sequence satisfying the condition $\left(\mathcal{A}_{\infty}\right)$ :

$$
\sum_{M<n \leq e^{M}} A_{n}=o(1) \quad \text { as } M \rightarrow \infty .
$$

Then for any $f \in C(\mathbb{T})$ with $a_{n}(f) \leq A_{n}$ for $n=1,2, \ldots$, we have

$$
\lim _{m \rightarrow \infty}\left\|f-G_{m}^{t}(f, \mathcal{T})\right\|_{\infty}=0 .
$$

The condition $\left(\mathcal{A}_{\infty}\right)$ is very close to the convergence of the series $\sum_{n} A_{n}$; if it holds then

$$
\sum_{n=1}^{N} A_{n}=o\left(\log _{*}(N)\right) \quad \text { as } N \rightarrow \infty,
$$

where $\log _{*}(u)$ is defined to be bounded for $u \leq 0$ and to satisfy $\log _{*}(u)=$ $\log _{*}(\log u)+1$ for $u>0$. The function $\log _{*}(u)$ grows more slowly than any iterated logarithmic function.

The condition $\left(\mathcal{A}_{\infty}\right)$ in Theorem 3 is sharp.

TheOREM 4. Assume that a decreasing sequence $\left\{A_{n}\right\}_{n=1}^{\infty}$ does not satisfy the condition $\left(\mathcal{A}_{\infty}\right)$. Then there exists a function $f \in C(\mathbb{T})$ with $a_{n}(f) \leq A_{n}$ for $n=1,2, \ldots$ and such that

$$
\limsup _{m \rightarrow \infty}\left\|f-G_{m}(f, \mathcal{T})\right\|_{\infty}>0
$$

for some realization $G_{m}(f, \mathcal{T})$.

Theorems 3 and 4 will be proved in Section 4. Also, in that section we will prove the following theorem.

THEOREM 5. Assume that a decreasing sequence $\left\{A_{n}\right\}_{n=1}^{\infty}$ is not summable. Then there exists a function $f \in C(\mathbb{T})$ with $a_{n}(f) \leq A_{n}$ for all $n$, such that the partial Fourier sums of $f$ diverge at some point. 
We note (see Section 2) that sufficient conditions for convergence of greedy approximants in Theorem 1 for $p=\infty$ also imply the convergence of partial Fourier sums. Theorems 3 and 5 demonstrate that the conditions for convergence of greedy approximants in terms of the decreasing rearrangements of the Fourier coefficients of continuous functions are weaker than the ones for convergence of partial Fourier sums.

2. Sufficient conditions in terms of Fourier coefficients. Proof of Theorem 1. Let us begin this section with some historical remarks. The question of the rate of greedy approximation of functions in certain smoothness classes was discussed in [T1]. In particular the following function class was considered. For $0<r<\infty$ and $0<q \leq \infty$, let $\mathcal{F}_{q}^{r}$ denote the class of those functions in $L_{1}\left(\mathbb{T}^{d}\right)$ such that

$$
|f|_{\mathcal{F}_{q}^{r}}:=\left\|\left(|k|^{r}|\widehat{f}(k)|\right)_{k \in \mathbb{Z}^{d}}\right\|_{l_{q}} \leq 1, \quad|\widehat{f}(0)| \leq 1 .
$$

Here we use the notation $|k|:=\max \left\{\left|k_{1}\right|, \ldots,\left|k_{d}\right|\right\}$. The following error estimates have been proved in [T1] for

$$
G_{m}\left(\mathcal{F}_{q}^{r}\right)_{p}:=\sup _{f \in \mathcal{F}_{q}^{r}}\left\|f-G_{m}(f)\right\|_{p} .
$$

Theorem 2.1. For any $0<q<\infty$ and $r>d(1-1 / q)_{+}$we have

$$
\begin{aligned}
G_{m}\left(\mathcal{F}_{q}^{r}\right)_{p} \asymp m^{-r / d-1 / q+1 / 2}, & & 1 \leq p \leq 2, \\
G_{m}\left(\mathcal{F}_{q}^{r}\right)_{p} \asymp m^{-r / d-1 / q+1-1 / p}, & & 2 \leq p \leq \infty .
\end{aligned}
$$

It has also been noticed in [T1] that the method used in the proof of Theorem 2.1 allows us to prove order estimates similar to (2.1) and (2.2) for classes a little wider than $\mathcal{F}_{q}^{r}$. We define these classes now. It is easy to verify that for $f \in \mathcal{F}_{q}^{r}$ and each $l \geq 1$ we have

$$
\left(\sum_{2^{l-1} \leq|k|<2^{l}}|\widehat{f}(k)|^{q}\right)^{1 / q} \leq 2^{-r(l-1)}, \quad|\widehat{f}(0)| \leq 1 .
$$

We use (2.3) as the definition of a new class $\mathcal{D F}_{q}^{r}$ ( $\mathcal{D}$ stands here to stress that restrictions are imposed on the dyadic blocks). Here is a remark from [T1].

Remark to Theorem 2.1. The relations (2.1) and (2.2) are valid when the class $\mathcal{F}_{q}^{r}$ is replaced by $\mathcal{D} \mathcal{F}_{q}^{r}$.

For $r>0$ and $0<q<\infty$, denote by $\mathcal{F} o_{q}^{r}$ the space of functions $f \in$ $L_{1}\left(\mathbb{T}^{d}\right)$ satisfying

$$
\sum_{|k|>n}|\widehat{f}(k)|^{q}=o\left(n^{-r q}\right) .
$$

We will now prove Theorem 1 from the introduction. 
Theorem 1. Let $2<p \leq \infty$ and $q>p^{\prime}=p /(p-1)$. Assume that $f \in L_{p}\left(\mathbb{T}^{d}\right) \cap \mathcal{F} o_{q}^{r}$ with $r=d\left(1 / p^{\prime}-1 / q\right)$. Then for any $0<t \leq 1$ we have

$$
\left\|f-G_{m}^{t}(f)\right\|_{p} \rightarrow 0 \quad \text { as } m \rightarrow \infty .
$$

Proof. First we note that (2.4) is equivalent to

$$
\sum_{k \in U(l)}|\widehat{f}(k)|^{q} \leq o\left(2^{-r l q}\right), \quad l=1,2, \ldots,
$$

where $U(l):=\left\{k \in \mathbb{Z}^{d}: 2^{l-1} \leq|k|<2^{l}\right\}$. It has been proved in [T1, (3.18)] that the estimates

$$
\sum_{k \in U(l)}|\widehat{f}(k)|^{q} \leq 2^{-r l q}, \quad l=1,2, \ldots
$$

imply

$$
a_{m}(f)=O\left(m^{-r / d-1 / q}\right) .
$$

In the same way one can prove that (2.5) implies that

$$
a_{m}(f)=o\left(m^{-r / d-1 / q}\right) .
$$

Since $r=d\left(1 / p^{\prime}-1 / q\right)$ we deduce from $(2.6)$ that

$$
a_{m}(f)=o\left(m^{-1 / p^{\prime}}\right) .
$$

In the case $2<p<\infty$ we can finish the proof of Theorem 1 by applying Theorem 2 from the introduction. However, we choose to give an independent proof for the following two reasons. The proof below is simpler than the proof of Theorem 2 (see Section 3); moreover, the proof below covers the case $p=\infty$, where Theorem 2 does not hold (see Section 4 ).

Let

$$
G_{m}^{t}(f)=S_{\Lambda_{m}}(f)
$$

with $\Lambda_{m}$ satisfying (1.1). Consider first the case $2<p<\infty$ and estimate $\left\|S_{m}^{d}(f)-S_{\Lambda_{m}}(f)\right\|_{p}$, where

$$
S_{m}^{d}(f):=\sum_{k \in Q(m)} \widehat{f}(k) e^{i(k, x)}, \quad Q(m):=\left\{k:|k| \leq m^{1 / d}\right\} .
$$

Then we have

$$
\begin{aligned}
S_{m}^{d}(f)-S_{\Lambda_{m}}(f) & =\sum_{k \in Q(m) \backslash \Lambda_{m}} \widehat{f}(k) e^{i(k, x)}-\sum_{k \in \Lambda_{m} \backslash Q(m)} \widehat{f}(k) e^{i(k, x)} \\
& =: \Sigma_{1}-\Sigma_{2} .
\end{aligned}
$$

From the definition of $\Lambda_{m}$ we get

$$
a_{m+1}(f) \leq \max _{k \notin \Lambda_{m}}|\widehat{f}(k)| \leq t^{-1} \min _{k \in \Lambda_{m}}|\widehat{f}(k)| \leq t^{-1} a_{m}(f) .
$$


Thus by the Hausdorff-Young theorem (see [Z, Chap. 12, Section 2]),

$$
\left\|\Sigma_{1}\right\|_{p} \leq\left(\sum_{k \in Q(m) \backslash \Lambda_{m}}|\widehat{f}(k)|^{p^{\prime}}\right)^{1 / p^{\prime}}=O\left(a_{m}(f) m^{1 / p^{\prime}}\right)=o(1) .
$$

Using the Hausdorff-Young theorem again and also the Hölder inequality with parameter $q / p^{\prime}$ we get

$$
\begin{aligned}
\left\|\Sigma_{2}\right\|_{p} & \leq\left(\sum_{k \in \Lambda_{m} \backslash Q(m)}|\widehat{f}(k)|^{p^{\prime}}\right)^{1 / p^{\prime}} \leq\left(\sum_{k \in \Lambda_{m} \backslash Q(m)}|\widehat{f}(k)|^{q}\right)^{1 / q} m^{1 / p^{\prime}-1 / q} \\
& \leq\left(\sum_{k \notin Q(m)}|\widehat{f}(k)|^{q}\right)^{1 / q} m^{1 / p^{\prime}-1 / q}=o(1) .
\end{aligned}
$$

It remains to remark that $\left\|f-S_{m}^{d}(f)\right\|_{p} \rightarrow 0$ as $m \rightarrow \infty$.

Let us now consider the case $p=\infty$. We remark that the relation (2.5) with $r=d(1-1 / q)$ and the Hölder inequality imply

$$
\sum_{n \leq|k|<2 n}|\widehat{f}(k)|=o(1) .
$$

First, observe that the cubic Fourier sums $S_{n}(f)$ uniformly converge to $f$ as $n \rightarrow \infty$. Indeed, consider the de la Vallée Poussin sums

$$
V_{n}(f)=\sum_{|k| \leq 2 n} \prod_{j=1}^{d} \min \left(1, \frac{2 n-\left|k_{j}\right|}{n}\right) \widehat{f}(k) e^{i(k, x)} .
$$

It is known (see [B]) that for any $f \in C\left(\mathbb{T}^{d}\right)$,

$$
\left\|V_{n}(f)-f\right\|_{\infty}=o(1) \quad(n \rightarrow \infty) .
$$

Further,

$$
\left\|S_{n}(f)-V_{n}(f)\right\|_{\infty} \leq \sum_{k \in \mathbb{Z}^{d}, n<|k| \leq 2 n}|\widehat{f}(k)|,
$$

and by (2.10),

$$
\left\|S_{n}(f)-V_{n}(f)\right\|_{\infty}=o(1) \quad(n \rightarrow \infty) .
$$

The relations (2.11) and (2.12) imply

$$
\left\|S_{n}(f)-f\right\|_{\infty}=o(1) \quad(n \rightarrow \infty) .
$$

Thus, we obtain the uniform convergence of $S_{n}(f)$ to $f$.

The rest of the proof is similar to the above case $2<p<\infty$ with the only difference that instead of the Hausdorff-Young theorem we use the inequality $\|f\|_{\infty} \leq \sum_{k}|\widehat{f}(k)|$. Theorem 1 is proved.

Let us now discuss the possibility of improving the assumption $f \in$ $L_{p}\left(\mathbb{T}^{d}\right) \cap \mathcal{F} o_{q}^{r}, r=d\left(1 / p^{\prime}-1 / q\right)$, in Theorem 1. 
Proposition 2.1. For each $2<p \leq \infty$ there exists $f \in L_{p}\left(\mathbb{T}^{d}\right)$ such that

$$
|\widehat{f}(k)|=O\left(|k|^{-d(1-1 / p)}\right)
$$

(and therefore $f \in \mathcal{D} \mathcal{F}_{q}^{r}$ with $\left.r=d\left(1 / p^{\prime}-1 / q\right)\right)$ and the sequence $\left\{G_{m}(f)\right\}$ diverges in $L_{p}$.

Proof. We will apply a construction from [T1]. We make use of the Rudin-Shapiro polynomials:

$$
\mathcal{R}_{N}(x)=\sum_{|k| \leq N} \varepsilon_{k} e^{i k x}, \quad \varepsilon_{k}= \pm 1, x \in \mathbb{T},
$$

which satisfy the estimate

$$
\left\|\mathcal{R}_{N}\right\|_{\infty} \leq C N^{1 / 2}
$$

for an absolute constant $C$. For $s= \pm 1$ define

$$
\Lambda_{ \pm 1}:=\left\{k: \widehat{\mathcal{R}}_{m}(k)= \pm 1\right\} .
$$

The estimate (2.17) implies

$$
|| \Lambda_{1}|-| \Lambda_{-1}||=\left|\mathcal{R}_{m}(0)\right| \leq C m^{1 / 2} .
$$

Let $s= \pm 1$ be such that $\left|\Lambda_{s}\right|>\left|\Lambda_{-s}\right|$. Take a small positive $\delta$ and consider the function

$$
f_{m, \delta}:=\mathcal{R}_{m}+s \delta \mathcal{D}_{m}
$$

where

$$
\mathcal{D}_{m}(x):=\sum_{|k| \leq m} e^{i k x}, \quad x \in \mathbb{T},
$$

is the Dirichlet kernel. Since $\left|\widehat{f}_{m, \delta}(k)\right|=1+\delta$ for $k \in \Lambda_{s}$ and $\left|\widehat{f}_{m, \delta}(k)\right|=1-\delta$ for $k \in \Lambda_{-s}$ and $\left|\Lambda_{s}\right| \geq m$, the frequencies of $G_{m}\left(f_{m, \delta}\right)$ are in $\Lambda_{s}$ and

$$
\left\|G_{m}\left(f_{m, \delta}\right)\right\|_{\infty} \geq\left|G_{m}\left(f_{m, \delta}\right)(0)\right|=(1+\delta) m .
$$

Next,

$$
\begin{aligned}
\left\|f_{m, \delta}\right\|_{p} & \leq\left\|\mathcal{R}_{m}\right\|_{p}+\delta\left\|\mathcal{D}_{m}\right\|_{p} \leq\left\|\mathcal{R}_{m}\right\|_{\infty}+\delta\left\|\mathcal{D}_{m}\right\|_{2}^{2 / p}\left\|\mathcal{D}_{m}\right\|_{\infty}^{1-2 / p} \\
& \leq C m^{1 / 2}+\delta(2 m+1)^{1-1 / p} \leq C_{1} m^{1 / 2}
\end{aligned}
$$

for $\delta \leq m^{1 / p-1 / 2}$. By the Nikol'skiı inequality for trigonometric polynomials the relation (2.20) implies

$$
\left\|G_{m}\left(f_{m, \delta}\right)\right\|_{p} \geq C_{2} m^{-1 / p}\left\|G_{m}\left(f_{m, \delta}\right)\right\|_{\infty} \geq C_{2} m^{1-1 / p} .
$$

Now define

$$
f_{m, \delta}^{d}(x):=\prod_{j=1}^{d} f_{m, \delta}\left(x_{j}\right) e^{i(4 m) x_{j}}
$$


and

$$
f:=\sum_{l=1}^{\infty} 2^{-d(1-1 / p) l} f_{2^{l}, \delta_{l}}^{d}(x), \quad 0<\delta_{l}<2^{-d l-3} .
$$

The relation (2.14) is obviously satisfied. Moreover, (2.21) implies that

$$
\left\|f-V_{2^{n}}(f)\right\|_{\infty}=O\left(2^{-d(1 / 2-1 / p) n}\right) .
$$

However, (2.22) shows that $\left\{G_{m}(f)\right\}$ diverges in $L_{p}$.

Let us make some more comments. For a given set $\Lambda$ define

$$
E_{\Lambda}(f)_{p}:=\inf _{c_{k}, k \in \Lambda}\left\|f-\sum_{k \in \Lambda} c_{k} e^{i(k, x)}\right\|_{p} .
$$

REMARK 2.1. Theorem 1 implies that if $f \in L_{p}, 2<p \leq \infty$, and

$$
E_{Q(n)}(f)_{2}=o\left(n^{-(1 / 2-1 / p)}\right)
$$

then $G_{m}^{t}(f) \rightarrow f$ in $L_{p}$.

Indeed, (2.24) is equivalent to $f \in \mathcal{F}_{o_{2}^{r}}$ with $r=d(1 / 2-1 / p)$.

REMARK 2.2. The proof of Proposition 2.1 (see (2.23)) implies that there is $f \in L_{p}\left(\mathbb{T}^{d}\right)$ such that

$$
E_{Q(n)}(f)_{\infty}=O\left(n^{1 / p-1 / 2}\right)
$$

and $\left\{G_{m}(f)\right\}$ diverges in $L_{p}, 2<p \leq \infty$.

REMARK 2.3. There exists a continuous function $f$ satisfying (2.10) such that $\left\{G_{m}(f)\right\}$ diverges in the uniform norm. with

We construct an example in the univariate case. Define $f:=\sum_{k \geq 2} b_{k}$

$$
b_{k}:=s_{k}^{-1 / 2} \sum_{l=1}^{s_{k}} 2^{-s_{k}} f_{2^{s_{k}}, \delta_{s_{k}}} e^{i 4^{s_{k}+l} x},
$$

where $\left\{s_{k}\right\}$ is an increasing sequence such that all frequencies of $b_{k+1}$ lie to the right of the frequencies of $b_{k}$. Then by (2.21) we get

$$
\left\|b_{k}\right\|_{\infty} \leq C_{1} s_{k}^{1 / 2} 2^{-s_{k} / 2}
$$

and therefore $f \in C(\mathbb{T})$. The relation (2.10) is also satisfied. It is clear that

$$
\max _{m}\left\|G_{m}\left(b_{k}\right)\right\|_{\infty} \geq s_{k}^{1 / 2} \text {. }
$$

This implies the divergence of $\left\{G_{m}(f)\right\}$.

REMARK 2.4. The construction in the proof of Proposition 2.1 can be used to prove that the convergence set for greedy approximation $\left\{G_{m}\right\}_{m=1}^{\infty}$ is not linear in $L_{p}, 2<p \leq \infty$. 
Indeed, consider

$$
g_{m, \delta}:=\sum_{|k| \leq m} \widehat{\mathcal{R}}_{m}(k)(1-\delta k / m) e^{i k x}, \quad h_{m, \delta}:=f_{m, \delta}-g_{m, \delta},
$$

where $f_{m, \delta}$ is defined by (2.19). Similarly to the definition of $f(d=1)$,

$$
f:=\sum_{l=1}^{\infty} 2^{-(1-1 / p) l} f_{2^{l}, \delta_{l}} e^{i 2^{l+2} x}, \quad 0<\delta_{l}<2^{-l-3},
$$

we define

$$
\begin{array}{ll}
g:=\sum_{l=1}^{\infty} 2^{-(1-1 / p) l} g_{2^{l}, \delta_{l}} e^{i 2^{l+2} x}, & 0<\delta_{l}<2^{-l-3}, \\
h:=\sum_{l=1}^{\infty} 2^{-(1-1 / p) l} h_{2^{l}, \delta_{l}} e^{i 2^{l+2} x}, & 0<\delta_{l}<2^{-l-3} .
\end{array}
$$

Thus $f=g+h$. It has been proved in Proposition 2.1 that the sequence $\left\{G_{m}(f)\right\}_{m=1}^{\infty}$ diverges in $L_{p}$. However, it is easy to check that $\left\{G_{m}(g)\right\}_{m=1}^{\infty}$ and $\left\{G_{m}(h)\right\}_{m=1}^{\infty}$ converge uniformly. Indeed, $h$ has an absolutely convergent Fourier series and $G_{m}(g)=S_{N}(g)$ for some $N$ (greedy ordering for $g$ coincides with the natural ordering). Then the uniform convergence of $\left\{G_{m}(g)\right\}_{m=1}^{\infty}$ follows from (2.23).

We note that the statement in Remark 2.1 can also be obtained from some general inequalities for $\left\|f-G_{m}(f)\right\|_{p}$. We now define the $m$-term best approximation to be

$$
\sigma_{m}(f)_{p}:=\inf _{k^{j} \in \mathbb{Z}^{d}, c_{j}}\left\|f-\sum_{j=1}^{m} c_{j} e^{i\left(k^{j}, x\right)}\right\|_{p} .
$$

It has been proved in [T1] that for any $f \in L_{p}\left(\mathbb{T}^{d}\right)$ one has

$$
\left\|f-G_{m}(f)\right\|_{p} \leq\left(1+3 m^{h(p)}\right) \sigma_{m}(f)_{p}, \quad 1 \leq p \leq \infty,
$$

where $h(p):=|1 / 2-1 / p|$. Similarly to the above inequality one can prove the following relation.

Theorem 2.2. For each $f \in L_{p}\left(\mathbb{T}^{d}\right)$ and any $0<t \leq 1$ we have

$$
\left\|f-G_{m}^{t}(f)\right\|_{p} \leq\left(1+(2+1 / t) m^{h(p)}\right) \sigma_{m}(f)_{p}, \quad 1 \leq p \leq \infty,
$$

where $h(p):=|1 / 2-1 / p|$.

Proof. We repeat the proof of Theorem 2.1 from [T1] that corresponds to the case $t=1$ with one minor change. Let

$$
G_{m}^{t}(f)=\sum_{k \in \Lambda^{\prime}(t)} \widehat{f}(k) e^{i(k, x)}, \quad\left|\Lambda^{\prime}(t)\right|=m, \quad \Lambda^{\prime}:=\Lambda^{\prime}(1) .
$$


Then the change in the proof from [T1] to adjust it to the case of $t<1$ is the following. Instead of the obvious relation (see [T1, (2.10)]):

$$
\left\|S_{\Lambda \backslash \Lambda^{\prime}}(f)\right\|_{2} \leq\left\|S_{\Lambda^{\prime} \backslash \Lambda}(f)\right\|_{2} \quad \text { for any } \Lambda \text { with }|\Lambda|=m,
$$

we make use of the inequality

$$
\left\|S_{\Lambda \backslash \Lambda^{\prime}(t)}(f)\right\|_{2} \leq t^{-1}\left\|S_{\Lambda^{\prime}(t) \backslash \Lambda}(f)\right\|_{2} \quad \text { for any } \Lambda \text { with }|\Lambda|=m,
$$
which follows easily from the definition of $\Lambda^{\prime}(t)$.

We now prove one more inequality.

Proposition 2.2. Let $2 \leq p \leq \infty$. Then for any $f \in L_{p}\left(\mathbb{T}^{d}\right)$ and any $Q$ with $|Q| \leq m$, we have

$$
\left\|f-G_{m}^{t}(f)\right\|_{p} \leq\left\|f-S_{Q}(f)\right\|_{p}+(3+1 / t)(2 m)^{h(p)} E_{Q}(f)_{2} .
$$

Proof. As above, let $G_{m}^{t}(f)=\sum_{k \in \Lambda^{\prime}(t)} \widehat{f}(k) e^{i(k, x)}$. Then

$$
\left\|f-G_{m}^{t}(f)\right\|_{p} \leq\left\|f-S_{Q}(f)\right\|_{p}+\left\|S_{Q}(f)-S_{\Lambda^{\prime}(t)}(f)\right\|_{p},
$$

and by [T1, Lemma 2.2], g

$$
\left\|S_{Q}(f)-S_{\Lambda^{\prime}(t)}(f)\right\|_{p} \leq(2 m)^{h(p)}\left\|S_{Q}(f)-S_{\Lambda^{\prime}(t)}(f)\right\|_{2} .
$$

Next,

$$
\left\|S_{Q}(f)-S_{\Lambda^{\prime}(t)}(f)\right\|_{2} \leq\left\|f-S_{Q}(f)\right\|_{2}+\left\|f-S_{\Lambda^{\prime}(t)}(f)\right\|_{2} .
$$

Using (2.25) with $\Lambda=\Lambda^{\prime}$ we get

$$
\begin{aligned}
\left\|S_{\Lambda^{\prime}(t)}(f)-S_{\Lambda^{\prime}}(f)\right\|_{2}^{2} & =\left\|S_{\Lambda^{\prime}(t) \backslash \Lambda^{\prime}}(f)\right\|_{2}^{2}+\left\|S_{\Lambda^{\prime} \backslash \Lambda^{\prime}(t)}(f)\right\|_{2}^{2} \\
& \leq\left(1+t^{-2}\right)\left\|S_{\Lambda^{\prime}(t) \backslash \Lambda^{\prime}}(f)\right\|_{2}^{2} \leq\left(1+t^{-2}\right) \sigma_{m}(f)_{2}^{2} .
\end{aligned}
$$

Therefore,

$$
\begin{aligned}
\left\|f-S_{\Lambda^{\prime}(t)}(f)\right\|_{2} & \leq\left\|f-S_{\Lambda^{\prime}}(f)\right\|_{2}+\left\|S_{\Lambda^{\prime}(t)}(f)-S_{\Lambda^{\prime}}(f)\right\|_{2} \\
& \leq(2+1 / t) \sigma_{m}(f)_{2} \leq(2+1 / t) E_{Q}(f)_{2} .
\end{aligned}
$$

Combining (2.26)-(2.29) we complete the proof of Proposition 2.2.

We now study the convergence of greedy approximations of univariate functions of bounded $\Phi$-variation. Let $\Phi: \mathbb{R}_{+} \rightarrow \mathbb{R}_{+}$be an increasing function and $\Phi(0)=0$. The class $V_{\Phi}$ of functions of bounded $\Phi$-variation is defined as the set of functions $f$ defined on $\mathbb{T}$ such that

$$
v_{\phi}(f)=\sup \sum_{j} \Phi\left(\left|f\left(b_{j}\right)-f\left(a_{j}\right)\right|\right)<\infty,
$$

where the supremum is taken over all possible finite systems of disjoint intervals $\left(a_{j}, b_{j}\right) \subset \mathbb{T}$. For $\Phi(u)=u$ the class $V_{\Phi}$ is the class of functions of bounded variation. Clearly, if $\Phi_{1}(u) \leq C \Phi_{2}(u)$, then $V_{\Phi_{2}} \subset V_{\Phi_{1}}$.

The classical Dirichlet-Jordan test asserts that if $f \in C(\mathbb{T})$ is a function of bounded variation then the Fourier series of $f$ uniformly converges to $f$ 
(see [Z, p. 57]). The convergence of Fourier series for functions of bounded $\Phi$-variation was studied by many authors; see related references in $[\mathrm{O}]$ where it was shown that the uniform convergence of Fourier series on the class $C(\mathbb{T}) \cap V_{\Phi}$ is equivalent to the condition

$$
\int_{0}^{1} \log (1 / \Phi(u)) d u<\infty .
$$

We proceed to a proposition that shows that we need a stronger restriction on $\Phi$ than the above one for convergence of greedy approximations.

Proposition 2.3. (a) If $u^{2}=o(\Phi(u))(u \rightarrow 0)$ and $f \in C(\mathbb{T}) \cap V_{\Phi}$, then

$$
\left\|f-G_{m}^{t}(f)\right\|_{\infty} \rightarrow 0 \quad \text { as } m \rightarrow \infty .
$$

(b) For $\Phi(u)=u^{2}$ there exists a function $f \in C(\mathbb{T}) \cap V_{\Phi}$ whose greedy approximants $\left\{G_{m}(f)\right\}$ diverge at $x=0$.

Proof. Let $1 \leq p \leq \infty, \delta>0$, and let $\omega(f, \delta)_{p}$ be the $L_{p}$ modulus of continuity of $f$ :

$$
\omega(f, \delta)_{p}=\sup _{0 \leq h \leq \delta}\|f(\cdot+h)-f(\cdot)\|_{p} .
$$

Let us estimate $\omega(f, \delta)_{2}$ for $f \in C(\mathbb{T}) \cap V_{\Phi}$. Take $h>0$ and $n=[2 \pi / h]+1$. We have

$$
\begin{aligned}
\| f(\cdot & +h)-f(\cdot) \|_{2}^{2} \\
& \leq \int_{0}^{n h}|f(t+h)-f(t)|^{2} d t=\sum_{j=1}^{n} \int_{(j-1) h}^{j h}|f(t+h)-f(t)|^{2} d t \\
& =\int_{0}^{h}\left(\sum_{j=1}^{n}|f(t+j h)-f(t+(j-1) h)|^{2}\right) d t \\
& =\int_{0}^{h} o\left(\sum_{j=1}^{n} \Phi(|f(t+j h)-f(t+(j-1) h)|)\right) d t=\int_{0}^{h} o\left(2 v_{\phi}(f)\right) d t=o(h) .
\end{aligned}
$$

Thus, $\omega(f, \delta)_{2}=o(\sqrt{\delta})$ as $\delta \rightarrow 0$, and, by Jackson's theorem [A, p. 200],

$$
E_{n}(f)_{2}=o\left(n^{-1 / 2}\right) \text {. }
$$

This means that $f$ satisfies (2.24) with $p=\infty$. By Remark 2.1, $\left\|f-G_{m}^{t}(f)\right\|_{\infty}$ $\rightarrow 0$ as $m \rightarrow \infty$.

To prove (b), we use the example from Remark 2.2 with $p=\infty$. We have $E_{n}(f)_{\infty}=O\left(n^{-1 / 2}\right)$. By Bernstein's theorem [A, p. 206], this implies $\omega(f, \delta)_{\infty} \leq C \sqrt{\delta}$ for some $C$. We show that the $\Phi$-variation of $f$ is finite for 
$\Phi(u)=u^{2}$. Indeed, for any disjoint intervals $\left(a_{j}, b_{j}\right)$,

$$
\sum_{j}\left|f\left(b_{j}\right)-f\left(a_{j}\right)\right|^{2} \leq \sum_{j} C^{2}\left|b_{j}-a_{j}\right| \leq 2 \pi C^{2},
$$

and so $v_{\phi}(f) \leq 2 \pi C^{2}$. This completes the proof of Proposition 2.3.

In particular, Proposition 2.3 implies that weak greedy approximations converge for any absolutely continuous function $f \in C(\mathbb{T})$. The same is true for $f \in C\left(\mathbb{T}^{2}\right)$. We use the notion of absolute continuity of a function of several variables suggested by L. Zajíček and developed in $[H]$. Let $\gamma \in(0,1)$. We say that a function $f: \mathbb{T}^{d} \rightarrow \mathbb{C}$ is absolutely continuous if for each $\varepsilon>0$ there is $\delta>0$ such that for each disjoint family $\left\{B_{j}:=B\left(t_{j}, r_{j}\right)\right\}$ of balls in $\mathbb{T}^{d}$ the inequality $\sum_{j} V\left(B_{j}\right)<\delta$ implies

$$
\sum_{j}\left(\sup _{t^{\prime}, t^{\prime \prime} \in B\left(t_{j}, \gamma r_{j}\right)}\left|f\left(t^{\prime}\right)-f\left(t^{\prime \prime}\right)\right|\right)^{d}<\varepsilon
$$

where $B(t, r)=\left\{t^{\prime}:\left|t-t^{\prime}\right| \leq r\right\}$ and $V(B)$ is the $d$-dimensional volume of the ball $B$. It is proven in $[\mathrm{H}]$ that the definition does not depend on $\gamma$ and for $d=1$ coincides with the classical definition.

Proposition 2.4. (a) If $f$ is absolutely continuous on $\mathbb{T}^{2}$, then we have $\left\|f-G_{m}^{t}(f)\right\|_{\infty} \rightarrow 0$ as $m \rightarrow \infty$.

(b) For $d>2$ there exists a function $f$ absolutely continuous on $\mathbb{T}^{d}$ such that its greedy approximants $\left\{G_{m}(f)\right\}$ diverge at $x=0$.

Proof. It is shown in $[\mathrm{H}]$ that the gradient of any absolutely continuous function $f \in C\left(\mathbb{T}^{d}\right)$ belongs to $L_{d}\left(\mathbb{T}^{d}\right)$. Therefore, in the case $d=2$ this implies $($ see $[\mathrm{N}])$ that

$$
E_{Q(n)}(f)_{2}=o\left(n^{-1 / 2}\right),
$$

and by Remark 2.1 we have

$$
\left\|f-G_{m}^{t}(f)\right\|_{\infty} \rightarrow 0 \quad \text { as } m \rightarrow \infty .
$$

Further, from the example in Remark 2.2 it is easy to see that for $d>2$ there exists a function $f$ continuously differentiable on $\mathbb{T}^{d}$ whose greedy approximations diverge at $x=0$. It follows from the definition that the class of absolutely continuous functions contains all continuously differentiable (and, moreover, all Lipschitzian) functions. This proves the proposition.

\section{Conditions in terms of decreasing rearrangements of Fourier} coefficients. Proof of Theorem 2. Let us begin with the proof of Theorem 2 . We repeat the statement for convenience.

Theorem 2. Let $2<p<\infty$ and let $\left\{A_{n}\right\}_{n=1}^{\infty}$ be a decreasing sequence satisfying

$$
A_{n}=o\left(n^{1 / p-1}\right) \quad \text { as } n \rightarrow \infty .
$$


Then for any $f \in L_{p}\left(\mathbb{T}^{d}\right)$ with $a_{n}(f) \leq A_{n}$ for $n=1,2, \ldots$, we have

$$
\lim _{m \rightarrow \infty}\left\|f-G_{m}^{t}(f, \mathcal{T})\right\|_{p}=0 .
$$

Proof. By the M. Riesz theorem (see [KS, Chap. 4, Section 3]), for any $f \in L_{p}\left(\mathbb{T}^{d}\right)$ with $1<p<\infty$ we have

$$
\left\|f-S_{N}(f)\right\|_{p} \rightarrow 0 \quad \text { as } N \rightarrow \infty .
$$

We first consider the case $t=1$. Let us estimate $\left\|S_{m}^{d}(f)-G_{m}(f)\right\|_{p}$. Set $\Sigma_{1}:=S_{m}^{d}\left(f-G_{m}(f)\right)$ and $\Sigma_{2}:=\left(\operatorname{Id}-S_{m}^{d}\right)\left(G_{m}(f)\right)$. Then

$$
S_{m}^{d}(f)-G_{m}(f)=S_{m}^{d}(f)-S_{m}^{d}\left(G_{m}(f)\right)-\left(\operatorname{Id}-S_{m}^{d}\right)\left(G_{m}(f)\right)=\Sigma_{1}-\Sigma_{2} .
$$

For the first sum, by the Paley theorem (see [Z, Chap. 12, Section 5]) we get

$$
\left\|\Sigma_{1}\right\|_{p} \leq C(p, d)\left(\sum_{n=1}^{2 m+1} a_{m}(f)^{p} n^{p-2}\right)^{1 / p}=O\left(a_{m}(f) m^{1-1 / p}\right)=o(1) .
$$

We now proceed to the second sum $\Sigma_{2}$. We first prove a general inequality.

Proposition 3.1. Let $2 \leq p<\infty$ and $u \in L_{p},\|u\|_{p} \neq 0$. Then for any $v \in L_{p}$ we have

$$
\|u\|_{p} \leq\|u+v\|_{p}+\left(\|u\|_{2 p-2} /\|u\|_{p}\right)^{p-1}\|v\|_{2} .
$$

Proof. Set $F:=\|u\|_{p}^{1-p} \bar{u}|u|^{p-2}$. Then $\|F\|_{p^{\prime}}=1$ and $\langle F, u\rangle=\|u\|_{p}$. Therefore,

$$
\|u\|_{p}=\langle F, u\rangle=\langle F, u+v\rangle-\langle F, v\rangle \leq\|u+v\|_{p}+\|F\|_{2}\|v\|_{2} .
$$

It remains to observe that $\|F\|_{2}=\left(\|u\|_{2 p-2} /\|u\|_{p}\right)^{p-1}$.

Lemma 3.1. Let $2 \leq p<\infty$. Let $f \in L_{p}\left(\mathbb{T}^{d}\right)$ and assume that $a_{n}(f)=$ $o\left(n^{1 / p-1}\right)$. Then

$$
\left\|\left(\mathrm{Id}-S_{m}^{d}\right)\left(G_{m}(f)\right)\right\|_{p}=o(1) .
$$

Proof. We use Proposition 3.1 with

$$
u:=\left(\operatorname{Id}-S_{m}^{d}\right)\left(G_{m}(f)\right), \quad v:=f-S_{m}^{d}(f)-u .
$$

Then

$$
\|v\|_{2} \leq\left\|f-G_{m}(f)\right\|_{2} \leq\left(\sum_{n>m} a_{n}(f)^{2}\right)^{1 / 2}=o\left(m^{1 / p-1 / 2}\right) .
$$

By the Paley theorem,

$$
\|u\|_{2 p-2}^{p-1}=O\left(\left(\sum_{n=1}^{m} a_{n}(f)^{2 p-2} n^{2 p-4}\right)^{1 / 2}\right)=o\left(m^{1 / 2-1 / p}\right) .
$$

Combining (3.5) and (3.6) and taking into account that $\|u+v\|_{p}=$ $\left\|f-S_{m}^{d}(f)\right\|_{p}=o(1)$, by Proposition 3.1 we conclude that $\|u\|_{p}=o(1)$. Lemma 3.1 is now proved. 
The required estimate $\left\|\Sigma_{2}\right\|_{p}=o(1)$ follows from Lemma 3.1. This together with (3.4) completes the proof of Theorem 2 in the case $t=1$. The general case $0<t \leq 1$ follows from the case $t=1$ and Lemma 3.2 below.

Lemma 3.2. Let $2 \leq p<\infty, t \in(0,1]$, and let $f \in L_{p}\left(\mathbb{T}^{d}\right)$ be such that $a_{n}(f)=o\left(n^{1 / p-1}\right)$. Then

$$
\left\|G_{m}(f)-G_{m}^{t}(f)\right\|_{p} \rightarrow 0 \quad \text { as } m \rightarrow \infty .
$$

Proof. Let $G_{m}(f)=S_{\Lambda}(f)$ and $G_{m}^{t}(f)=S_{\Lambda(t)}(f)$. Then

$$
g_{m}:=G_{m}(f)-G_{m}^{t}(f)=\sum_{k \in \Lambda \backslash \Lambda(t)} \widehat{f}(k) e^{i(k, x)}-\sum_{k \in \Lambda(t) \backslash \Lambda} \widehat{f}(k) e^{i(k, x)} .
$$

It is clear that

$$
|\widehat{f}(k)| \leq a_{m}(f), \quad k \in \Lambda(t) \backslash \Lambda .
$$

The relation (2.8) implies

$$
|\widehat{f}(k)| \leq t^{-1} a_{m}(f), \quad k \in \Lambda \backslash \Lambda(t) .
$$

Thus, for the Fourier coefficients of the function $g_{m}$ we have

$$
\left|\widehat{g}_{m}(k)\right| \leq t^{-1} a_{m}(f) .
$$

Taking into account that $g_{m}$ has at most $2 m$ terms, the Paley theorem shows that

$$
\left\|g_{m}\right\|_{p}=O\left(a_{m}(f) m^{1-1 / p}\right)=o(1) .
$$

This proves the lemma.

Let us note that by the Hausdorff-Young theorem the condition

$$
\sum_{n=1}^{\infty} A_{n}^{p^{\prime}}<\infty, \quad 2<p<\infty,
$$

which is stronger than (3.1), implies that for any $f$ such that $a_{n}(f) \leq A_{n}$ its Fourier series converges in $L_{p}$ unconditionally.

Proposition 3.2. Let $2<p<\infty$. Suppose that a decreasing sequence $\left\{A_{n}\right\}_{n=1}^{\infty}$ does not satisfy the condition (3.1) of Theorem 2, i.e.,

$$
\limsup _{n \rightarrow \infty} A_{n} n^{1-1 / p}>0 .
$$

Then there is $f \in C(\mathbb{T})$ with $a_{n}(f) \leq A_{n}$ for $n=1,2, \ldots$ such that $\left\{G_{m}(f)\right\}$ diverges in $L_{p}$.

Proof. We make use of the functions constructed in the proof of Proposition 2.1. Let $c>0$ and $\left\{n_{k}\right\}$ be such that

$$
A_{n_{k}} \geq c n_{k}^{1 / p-1}, \quad n_{k} \geq 4 n_{k-1}, \quad n_{1} \geq 4 .
$$


Define $m_{k}:=\left[n_{k} / 4\right]$ and

$$
f:=c \sum_{k=1}^{\infty} n_{k}^{1 / p-1} f_{m_{k}, \delta_{k}} e^{i n_{k} x},
$$

where the $f_{m, \delta}$ are defined by (2.19). Then $f$ is a continuous function satisfying $a_{n}(f) \leq A_{n}$. The divergence of $\left\{G_{m}(f)\right\}$ follows from (2.22).

\section{Conditions in terms of decreasing rearrangements of Fourier} coefficients. Proof of Theorems 3-5. We begin with the proof of Theorem 3 . We reformulate it here for convenience.

THEOREM 3. Let $\left\{A_{n}\right\}_{n=1}^{\infty}$ be a decreasing sequence satisfying the condition $\left(\mathcal{A}_{\infty}\right)$ :

$$
\sum_{M<n \leq e^{M}} A_{n}=o(1) \quad \text { as } M \rightarrow \infty .
$$

Then for any $f \in C(\mathbb{T})$ with $a_{n}(f) \leq A_{n}$ for $n=1,2, \ldots$, we have

$$
\lim _{m \rightarrow \infty}\left\|f-S_{\Lambda_{m}}(f)\right\|_{\infty}=0
$$

where $\Lambda_{m}$ is an arbitrary subset of $\mathbb{Z}$ satisfying

$$
\begin{gathered}
\left|\Lambda_{m}\right|=m, \\
\min _{k \in \Lambda_{m}}|\widehat{f}(k)| \geq t \max _{k \notin \Lambda_{m}}|\widehat{f}(k)| .
\end{gathered}
$$

Proof. Set as above

$$
G_{m}(f)=\sum_{n=1}^{m} \widehat{f}(k(n)) e^{i k(n) x} .
$$

Note that if $k \neq k(n)$ for $n \leq m$ then $|\widehat{f}(k)| \leq a_{m}(f)$. Also, by (4.4), if $k \notin \Lambda_{m}$ then $|\widehat{f}(k)| \leq a_{m}(f) / t$. Therefore,

$$
\left\|S_{\Lambda_{m}}(f)-G_{m}(f)\right\|_{\infty} \leq m a_{m}(f)+m a_{m}(f) / t .
$$

It is clear that (4.1) implies $A_{n}=o\left(n^{-1}\right)$, and therefore

$$
a_{m}(f) m=o(1) \text {. }
$$

Relations (4.5) and (4.6) give

$$
\left\|S_{\Lambda_{m}}(f)-G_{m}(f)\right\|_{\infty}=o(1) .
$$

Let us estimate $\left\|V_{m}(f)-G_{m}(f)\right\|_{\infty}$, where $V_{m}(f)$ is the de la Vallée Poussin sum

$$
V_{m}(f)=\sum_{|k| \leq 2 m} \min \left(1, \frac{2 m-|k|}{m}\right) \widehat{f}(k) e^{i k x} .
$$


We have $V_{m}(f)-G_{m}(f)=\Sigma_{1}-\Sigma_{2}$, where

$$
\Sigma_{1}=V_{m}\left(f-G_{m}(f)\right), \quad \Sigma_{2}=\left(\operatorname{Id}-V_{m}\right)\left(G_{m}(f)\right) .
$$

For the first sum we get

$$
\left\|\Sigma_{1}\right\|_{\infty} \leq \sum_{n=1}^{4 m-1} a_{m}(f) \leq 4 m a_{m}(f)
$$

Therefore, by (4.6), $\left\|\Sigma_{1}\right\|_{\infty}=o(1)$.

We proceed to the second sum $\Sigma_{2}$. Consider

$$
f-V_{m}(f)-\Sigma_{2}=\sum_{m<n \leq e^{e^{m}}, k(n)>m} \lambda_{n} \widehat{f}(k(n)) e^{i k(n) x}+g=: \Sigma_{3}+g,
$$

where $0 \leq \lambda_{n} \leq 1$. Using (2.11) and the assumption $\left(\mathcal{A}_{\infty}\right)$ from (4.8) we get

$$
\left\|\Sigma_{2}+g\right\|_{\infty} \leq\left\|f-V_{m}(f)\right\|_{\infty}+\left\|\Sigma_{3}\right\|_{\infty}=o(1) .
$$

Next we have

$$
\|g\|_{2} \leq\left(\sum_{n>e^{e^{m}}} a_{n}(f)^{2}\right)^{1 / 2}=o\left(e^{-e^{m} / 2}\right) .
$$

We need the following lemma that we will prove a little later.

LEMMA 4.1. Suppose that a function $f$ with $\|f\|_{\infty}=1$ has the form

$$
f=\sum_{k \in \Lambda} \widehat{f}(k) e^{i k x}, \quad|\Lambda| \leq m .
$$

Then for any function $g$ such that $\|g\|_{2} \leq \frac{1}{4}(4 \pi m)^{-m / 2}$ we have

$$
\|f+g\|_{\infty} \geq 1 / 4 \text {. }
$$

This lemma and (4.9) imply that $\left\|\Sigma_{2}\right\|_{\infty}=o(1)$. Together with (4.7) this completes the proof of Theorem 3.

Proof of Lemma 4.1. Denote by $\|u\|$ the distance from a real number $u$ to the closest integer. For a fixed $j \in \mathbb{N}$ define

$$
F_{j}=\{x \in \mathbb{T}: \forall k \in \Lambda,\|j(k x /(2 \pi))\|<1 /(4 \pi m)\}, \quad F=F_{1} .
$$

Well known estimates for simultaneous diophantine approximation (see [C, p. 13]) give

$$
\mathbb{T}=\bigcup_{j \leq J} F_{j}, \quad J=(4 \pi m)^{m} .
$$

Note that $\mu F_{j}=\mu F$ for all $j$. Therefore,

or

$$
1 \leq \sum_{j \leq J} \mu F_{j} \leq J \mu F,
$$




$$
\mu F \geq(4 \pi m)^{-m} .
$$

Let $\left|f\left(x_{0}\right)\right|=\|f\|_{\infty}=1$ and $E=\left\{x_{0}+y: y \in F\right\}$. For $x=x_{0}+y \in E$ and $k \in \Lambda$ we have

$$
\left|e^{i k x}-e^{i k x_{0}}\right| \leq 2 \pi\|k y /(2 \pi)\|<1 /(2 m)
$$

Therefore,

$$
\left|f(x)-f\left(x_{0}\right)\right| \leq \sum_{k \in \Lambda}|\widehat{f}(k)|\left|e^{i k x}-e^{i k x_{0}}\right| \leq \sum_{k \in \Lambda} 1 /(2 m) \leq 1 / 2 .
$$

Thus, $|f(x)| \geq 1 / 2$ for $x \in E$.

Suppose that

$$
\|f+g\|_{\infty}<1 / 4
$$

Then $|g(x)|>1 / 4$ for $x \in E$, and by (4.11),

$$
\|g\|_{2}^{2} \geq \int_{E}|g(x)|^{2} d \mu>\left(\frac{1}{4}\right)^{2}(4 \pi m)^{-m} .
$$

This contradicts the assumption of the lemma. Hence, (4.12) is not true, and the proof is complete.

REMARK 4.1. Actually, in the proof of Lemma 4.1 we have shown the following. If

$$
f=\sum_{k \in \Lambda} \widehat{f}(k) e^{i k x}, \quad|\Lambda| \leq m,
$$

and $G \subset \mathbb{T}$ has $\mu G>1-(4 \pi m)^{-m}$, then

$$
\|f\|_{\infty} \leq 2 \sup _{x \in G}|f(x)| .
$$

Recently the first author and Nazarov have proved (unpublished) that the last inequality holds under the assumption $\mu G>1-c^{m}$ for a small constant $c$. This can be used to weaken the assumption on $\|g\|_{2}$ in Lemma 4.1. However, it does not affect Theorem 3.

We proceed to the proof of Theorem 4 from the introduction. The core of the proof is the following lemma.

Lemma 4.2. Fix $\Delta, \delta>0$. Assume that positive integers $m \rightarrow \infty$ and $M \rightarrow \infty$ are such that

$$
\log M=o(m) .
$$

Let $m_{1}=m, m_{3}=m+M, m_{1}<m_{2}<m_{3}$. Let $\left\{A_{n}\right\}_{n=1}^{\infty}$ be a decreasing 
sequence satisfying

$$
\begin{aligned}
A_{n} & \leq \Delta / n, \\
\sum_{n=m_{1}+1}^{m_{2}} A_{n} & =\sum_{n=m_{2}+1}^{m_{3}} A_{n}=1, \\
A_{2 m} & >\delta A_{m} .
\end{aligned}
$$

Then for sufficiently large $m$ there exists a trigonometric polynomial $T(x)=$ $T_{m}(x)=\sum_{k=1}^{M} \widehat{T}(k) e^{i k x}$ such that

$$
\begin{array}{ll}
a_{k}(T) \leq A_{m+k} & (1 \leq k \leq M), \\
\|T\|_{\infty} \rightarrow 0 & (m \rightarrow \infty), \\
\max _{n}\left|G_{n}(T, \mathcal{T})(0)\right|>0.01
\end{array}
$$

Proof. Take independent random variables $\eta_{k}(1 \leq k \leq M)$ so that each $\eta_{k}$ takes value $n, m_{1}<n \leq m_{3}$, with probability $1 /(10 M)$, and $\eta_{k}=m_{1}$ with probability 0.9 . A polynomial $T$ is defined as

$$
T(x)=\sum_{k=1}^{M} \sigma_{\eta_{k}} A_{\eta_{k}} e^{i k x}
$$

where $\sigma\left(m_{1}\right)=0, \sigma_{n}=1$ for $m_{1}<n \leq m_{2}$, and $\sigma_{n}=-1$ for $m_{2}<n \leq m_{3}$. We prove that $T$ satisfies (4.17)-(4.19) with large probability. Probability, expectation and variance will be denoted by $P, E$, and $V$, respectively. We will estimate the probabilities of the following events:

$$
\begin{array}{ll}
E_{1}: & \exists l \geq 1:\left|\left\{k: m_{1}<\eta_{k} \leq m_{1}+l\right\}\right|>l \\
E_{2}: & \|T\|_{\infty}>3\left(A_{m} \log \left(2 \pi M^{2}\right)\right)^{1 / 2} \\
E_{3}: & \sum_{k: m_{1}<\eta_{k} \leq m_{2}} A_{\eta_{k}} \leq 0.05 .
\end{array}
$$

Note that nonoccurrence of $E_{1}, E_{2}, E_{3}$ implies (4.17), (4.18), (4.19), respectively. In the case of $E_{2}$ and (4.18) we apply (4.13) and (4.14) to prove that $A_{m} \log \left(2 \pi M^{2}\right)=o(1)$.

Consider the event

$$
E_{1, l}: \quad\left|\left\{k: m_{1}<\eta_{k} \leq m_{1}+l\right\}\right|>l .
$$

We have

$$
P\left(E_{1}\right) \leq \sum_{l} P\left(E_{1, l}\right) .
$$

Further, 


$$
\begin{aligned}
P\left(E_{1, l}\right) & =\sum_{j=l+1}^{M}\left(\begin{array}{c}
M \\
j
\end{array}\right)\left(\frac{l}{10 M}\right)^{j}\left(1-\frac{l}{10 M}\right)^{M-j} \\
& \leq \sum_{j=l+1}^{M}\left(\begin{array}{c}
M \\
j
\end{array}\right)\left(\frac{l}{10 M}\right)^{j} .
\end{aligned}
$$

For any $j>l$ we have

$$
\left(\frac{l}{10 M}\right)^{j}<10^{-l}\left(\frac{l}{M}\right)^{j}, \quad 1 \leq e^{l}\left(1-\frac{l}{M}\right)^{M-l} \leq e^{l}\left(1-\frac{l}{M}\right)^{M-j} .
$$

Therefore,

$$
\begin{aligned}
\sum_{j=l+1}^{M}\left(\begin{array}{c}
M \\
j
\end{array}\right)\left(\frac{l}{10 M}\right)^{j} & \leq(e / 10)^{l} \sum_{j=l+1}^{M}\left(\begin{array}{c}
M \\
j
\end{array}\right)\left(\frac{l}{M}\right)^{j}\left(1-\frac{l}{M}\right)^{M-j} \\
& \leq(e / 10)^{l} .
\end{aligned}
$$

By (4.20) and (4.21) we get

$$
P\left(E_{1}\right) \leq \sum_{l}(e / 10)^{l}<1 / 2 .
$$

To estimate $P\left(E_{2}\right)$, we use the following theorem [Ka, pp. 68, 79].

Theorem A. Let $E$ be a measurable space with measure $\mu$ and $\mu(E)<\infty$. Let $B$ be a linear space of measurable bounded functions on $E$, closed under complex conjugation, and suppose that there exists $\varrho>0$ with the following property: if $f \in B$ and $f$ is real, then there exists a measurable set $I=$ $I(f) \subset E$ such that $\mu(I)>\mu(E) / \varrho$ and $|f(t)| \geq \frac{1}{2}\|f\|_{\infty}$ for $t \in I$. Consider a random finite sum

$$
P=\sum \xi_{k} f_{k}
$$

where

$$
E\left(\xi_{k}\right)=0, \quad E\left(\xi_{k}^{2}\right)=b_{k}^{2}, \quad\left|\xi_{k}\right| \leq 1 .
$$

Moreover, suppose that $\left\|f_{k}\right\|_{\infty}=1$ and $r=\sum b_{k}^{2}>\log \varrho$. Then

$$
P\left(\|P\|_{\infty} \geq 6(r \log \varrho)^{1 / 2}\right) \leq 4 / \varrho .
$$

We apply Theorem A for $E=\mathbb{T}, B=\left\{\sum_{k=1}^{M} c_{k} e^{i(k, x)}\right\}, f_{k}=e^{i(k, x)}$, $\xi_{k}=\sigma_{\eta_{k}} A_{\eta_{k}} / A_{m}$. Note that

$$
P(x)=T(x) / A_{m} .
$$

One can guarantee the existence of the required set $I(f)$ by taking

$$
\varrho=2 \pi M^{2}
$$


([Ka, p. 49]). Further, for $k=1, \ldots, M$ we have $E \xi_{k}=0$, and by (4.16),

$$
b_{k}^{2}=E \xi_{k}^{2}=\frac{1}{10 M A_{m}^{2}} \sum_{n=m_{1}+1}^{m_{3}} A_{n}^{2} \geq \frac{m \delta^{2}}{10 M} .
$$

Therefore, $r>m \delta^{2} / 10$, and by (4.13) and (4.24), for sufficiently large $m$ the condition $r>\log \varrho$ holds. On the other hand,

$$
\sum_{n=m_{1}+1}^{m_{3}} A_{n}^{2} \leq A_{m} \sum_{n=m_{1}+1}^{m_{3}} A_{n}=2 A_{m}
$$

$b_{k}^{2} \leq 1 /\left(5 M A_{m}\right)$, and $r \leq 1 /\left(5 A_{m}\right)$. Thus, by $(4.23)$,

$$
\begin{aligned}
P\left(\|P\|_{\infty} \geq 6(r \log \varrho)^{1 / 2}\right) & \geq P\left(\|P\|_{\infty} \geq 3\left(\log \left(2 \pi M^{2}\right) / A_{m}\right)^{1 / 2}\right) \\
& =P\left(\|T\|_{\infty} \geq 3\left(A_{m} \log \left(2 \pi M^{2}\right)\right)^{1 / 2}\right),
\end{aligned}
$$

and Theorem A gives

$$
P\left(E_{2}\right) \leq 4 / \varrho \leq M^{-2} .
$$

To estimate $P\left(E_{3}\right)$, we define the random variables $\nu_{1}, \ldots, \nu_{M}$ as $\nu_{k}=$ $A_{\eta_{k}}$ for $m_{1}<\eta_{k} \leq m_{2}$ and $\nu_{k}=0$ otherwise. The event $E_{3}$ can be rewritten as

$$
E_{3}: \quad \sum_{k=1}^{M} \nu_{k} \leq 0.05 .
$$

We have $E\left(\nu_{k}\right)=1 /(10 M)$, and by $(4.25)$,

$$
V\left(\nu_{k}\right) \leq E\left(\nu_{k}^{2}\right) \leq \frac{A_{m}}{5 M} .
$$

Hence,

$$
E\left(\sum_{k=1}^{M} \nu_{k}\right)=0.1, \quad V\left(\sum_{k=1}^{M} \nu_{k}\right) \leq \frac{A_{m}}{5},
$$

and by Chebyshev's inequality,

$$
P\left(E_{3}\right) \leq \frac{V\left(\sum_{k=1}^{M} \nu_{k}\right)}{\left(E\left(\sum_{k=1}^{M} \nu_{k}\right)-0.05\right)^{2}} \leq 80 A_{m} .
$$

So, by (4.22), (4.26), and (4.27), $P\left(E_{1}\right)+P\left(E_{2}\right)+P\left(E_{3}\right)<1$, and there is a choice of $T$ for which none of $E_{1}, E_{2}, E_{3}$ holds. This completes the proof of Lemma 4.2.

TheOREM 4. Assume that a decreasing sequence $\left\{A_{n}\right\}_{n=1}^{\infty}$ does not satisfy the condition $\left(\mathcal{A}_{\infty}\right)$. Then there exists a function $f \in C(\mathbb{T})$ with $a_{n}(f) \leq A_{n}$ for $n=1,2, \ldots$ such that

$$
\limsup _{m \rightarrow \infty}\left\|f-G_{m}(f, \mathcal{T})\right\|_{\infty}>0 .
$$


Proof. Without loss of generality we may suppose that

$$
\limsup _{u \rightarrow \infty} \sum_{u<n \leq e^{u}} A_{n}>8
$$

where $u \in \mathbb{R}$. Also, we may assume that for sufficiently large $n$,

$$
A_{n} \leq 10 / n \text {. }
$$

Indeed, if (4.29) fails for infinitely many $n$ 's, we replace all $A_{n}$ by $A_{n}^{\prime}=$ $\min \left(A_{n}, 10 / n\right)$. If $A_{m}>10 / m$ for some large $m$, then

$$
\sum_{\log m<n \leq m} A_{n}^{\prime} \geq \sum_{\log m<n \leq m} 10 / m>9,
$$

and (4.28) holds for $A_{n}^{\prime}$. Now, observe that $F(F(u))>e^{u}$ for sufficienly large $u$ and $F(u)=e^{\sqrt{u}}$. Therefore,

$$
\sum_{u<n \leq e^{u}} A_{n} \leq \sum_{u<n \leq F(u)} A_{n}+\sum_{F(u)<n \leq F(F(u))} A_{n},
$$

and (4.28) implies

$$
\limsup _{u \rightarrow \infty} \sum_{u<n \leq e^{\sqrt{u}}} A_{n}>4 .
$$

We now prove that there exists an arbitrarily large integer $m$ such that

$$
\begin{gathered}
\sum_{\substack{m<n \leq e^{3 \sqrt{m}} \\
A_{2 m}}} A_{n}>3, \\
A_{m} / 100 .
\end{gathered}
$$

Indeed, by (4.30), we can take a large $u$ with

$$
\sum_{u<n \leq e \sqrt{u}} A_{n}>4 .
$$

Let $m_{0}=[u]$. If $A_{m_{0}} \geq 1 /\left(2 m_{0}\right)$, then $m=\left[m_{0} / 2\right]$ satisfies (4.31) and (4.32) (we use (4.29) with $n=m$ ). If $A_{m_{0}}<1 /\left(2 m_{0}\right)$, we define $m_{j}=2^{j} m_{0}$. We take for $m$ the minimal $m_{j}$ satisfying (4.32). To show the existence of such an $m$ and to prove (4.31), we note that

$$
\sum_{m_{0}<n \leq m_{j}} A_{n}<1
$$

whenever $A_{m_{1}}<A_{m_{0}} / 100, \ldots, A_{m_{j}}<A_{m_{j-1}} / 100$. Hence, the number $m$ does exist, and moreover,

$$
\sum_{m<n \leq e \sqrt{u}} A_{n}>3
$$

which clearly implies (4.31). 
We now take any large $m=m_{1}$ satisfying (4.31) and (4.32) and define

$$
\begin{aligned}
& m_{2}=\min \left\{m^{\prime}: \sum_{m_{1}<n \leq m^{\prime}} A_{n} \geq 1\right\}, \\
& m_{3}=\min \left\{m^{\prime}: \sum_{m_{2}<n \leq m^{\prime}} A_{n} \geq \sum_{m_{1}<n \leq m_{2}} A_{n}\right\} .
\end{aligned}
$$

We have

$$
\sum_{m_{1}<n \leq m_{3}} A_{n}<2+2 a_{m_{2}}+a_{m_{3}}<3 .
$$

This inequality combined with (4.31) shows that $m_{3}<e^{3 \sqrt{m}}$. We now apply Lemma 4.2 to the sequence $\left\{A_{n}^{\prime}\right\}$, where

$$
A_{n}^{\prime}= \begin{cases}\left(\sum_{m_{1}<k \leq m_{2}} A_{k}\right)^{-1} A_{n} & \left(n \leq m_{2}\right), \\ \left(\sum_{m_{2}<k \leq m_{3}} A_{k}\right)^{-1} A_{n} & \left(n>m_{2}\right) .\end{cases}
$$

We get a polynomial $T=T_{m}$ satisfying (4.17)-(4.19). Set

$$
f(x)=\sum_{m} T_{m}(x) e^{i n_{m} x}
$$

where the sum is taken over a sparse sequence of $m$ 's with $n_{m}$ chosen to make the sets of frequencies of $T_{m}(x) e^{i n_{m} x}$ disjoint. This completes the proof of Theorem 4 .

THEOREM 5. Assume that a decreasing sequence $\left\{A_{n}\right\}_{n=1}^{\infty}$ is not summable. Then there exists $f \in C(\mathbb{T})$ with $a_{n}(f) \leq A_{n}$ such that the partial Fouries sums diverge at some point.

Theorem 5 is a simple corollary of the following lemma.

Lemma 4.3. Assume that a decreasing sequence $\left\{A_{n}\right\}_{n=1}^{\infty}$ is not summable. Then for any $l \in \mathbb{N}$ and $m_{0} \in \mathbb{N}$ there exist a trigonometric polynomial $T(x)=T_{l}(x)$ and numbers $m \geq m_{0}, N \in \mathbb{N}$ such that

$$
\begin{array}{ll}
a_{k}(T) \leq A_{m+k} & (k \geq 1), \\
\|T\|_{\infty} \rightarrow 0 & (l \rightarrow \infty), \\
\left|S_{N}(T, 0)\right| \rightarrow \infty & (l \rightarrow \infty) .
\end{array}
$$

Proof of Lemma 4.3. By the conditions on $\left\{A_{n}\right\}$, for any $l \in \mathbb{N}$ we have $\sum_{n} A_{2 l n}=\infty$. Therefore, for any $l>1$ we can find $m_{1}>m_{0}$ and $m_{2}>m_{1}$ such that

$$
(\log l)^{-1 / 2} \leq \sum_{m_{1}<n \leq m_{2}} A_{2 l n} \leq 2(\log l)^{-1 / 2}
$$


We associate with any $n, m_{1}<n \leq m_{2}$, a trigonometric polynomial

where $K$ and $k_{n}$ satisfy

$$
T_{n}(x)=A_{2 l n} e^{i k_{n} x} \sum_{j=1}^{l} \frac{\sin (K j x)}{j},
$$

$$
k_{n}=N-n, \quad K>m_{2}, \quad N>l K
$$

for some positive integer $N$. We define

$$
T=\sum_{m_{1}<n \leq m_{2}} T_{n}
$$

Let us prove (4.33) with $m=2 m_{1}$. We observe that by the choice of $k_{n}$ and $K$ the spectra of $T_{n}$ are disjoint, that is, for any $j$ there exists at most one $n$ such that $\widehat{T}_{n}(j) \neq 0$. We have

$$
\begin{gathered}
T_{n}(x)=\sum_{1 \leq|j| \leq l} \widehat{T}_{n}\left(k_{n}+K j\right) e^{i\left(k_{n}+K j\right) x}, \\
\left|\widehat{T}_{n}\left(k_{n}+K j\right)\right|=A_{2 l n} /(2|j|) .
\end{gathered}
$$

Therefore,

$$
\begin{array}{ll}
\left|\widehat{T}_{n}\left(k_{n}+K j\right)\right| \leq A_{2 l n} \leq A_{2 l n-j} & (1 \leq j \leq l), \\
\left|\widehat{T}_{n}\left(k_{n}-K j\right)\right| \leq A_{2 l n} \leq A_{2 l n-n-j} & (1 \leq j \leq l) .
\end{array}
$$

Note that for $n>m_{1}, 1 \leq j \leq l$, the numbers $2 l n-j, 2 l n-n-j$ are all greater than $2 m_{1}$ and pairwise distinct. This proves (4.33) with $m=2 m_{1}$.

We now check (4.34) and (4.35). Using the well known estimate

$$
\left\|\sum_{j=1}^{l} \frac{\sin (j u)}{j}\right\|_{\infty} \leq C
$$

[Z, p. 61], we get

$$
\|T\|_{\infty} \leq C \sum_{m_{1}<n \leq m_{2}} A_{2 l n}
$$

and by (4.36),

$$
\|T\|_{\infty} \leq 2 C(\log l)^{-1 / 2}
$$

Let us estimate $S_{N}(T, 0)$. We have

Hence,

$$
S_{N}\left(T_{n}, 0\right)=\frac{i}{2} A_{2 l n} \sum_{j=1}^{l} \frac{1}{j} .
$$

$$
\left|S_{N}(T, 0)\right|=\frac{1}{2} \sum_{m_{1}<n \leq m_{2}} A_{2 l n} \sum_{j=1}^{l} \frac{1}{j},
$$

and (4.35) follows from (4.36). The proof is complete. 


\section{References}

[A] N. I. Achieser, Theory of Approximation, Ungar, New York, 1956.

[B] S. P. Baĭborodov, Approximation of functions of several variables by de la Vallée Poussin rectangular sums, Math. Notes 29 (1981), 362-372.

[C] J. W. S. Cassels, An Introduction to Diophantine Approximation, Cambridge Tracts in Math. and Math. Phys., Cambridge, 1957.

[CF] A. Córdoba and P. Fernández, Convergence and divergence of decreasing rearranged Fourier series, SIAM J. Math. Anal. 29 (1998), 1129-1139.

[D] R. A. DeVore, Nonlinear approximation, Acta Numer. 7 (1998), 51-150.

$[\mathrm{H}] \quad$ S. Hencl, On the notions of absolute continuity for functions of several variables, Fund. Math. 173 (2002), 175-189.

[K1] T. W. Körner, Divergence of decreasing rearranged Fourier series, Ann. of Math. 144 (1996), 167-180.

[K2] -, Decreasing rearranged Fourier series, J. Fourier Anal. Appl. 5 (1999), 1-19.

[Ka] J.-P. Kahane, Some Random Series of Functions, Cambridge Univ. Press, Cambridge, 1985.

[KBR] N. J. Kalton, N. T. Peck and J. W. Roberts, An F-space Sampler, London Math. Soc. Lecture Note Ser. 89, Cambridge Univ. Press, Cambridge, 1984.

[KS] B. S. Kashin and A. A. Saakyan, Orthogonal Series, Amer. Math. Soc., Providence, RI, 1989.

[N] S. N. Nikol'skiǔ, Approximation of Functions of Several Variables and Embedding Theorems, Springer, 1975 (English translation of the Russian edition published by "Nauka", Moscow, 1969).

[O] K. I. Oskolkov, Generalized variation, the Banach indicatrix and the uniform convergence of Fourier series, Math. Notes 12 (1972), 619-625.

[T1] V. N. Temlyakov, Greedy algorithm and $m$-term trigonometric approximation, Constr. Approx. 107 (1998), 569-587.

[T2] -, Nonlinear methods of approximation, Found. Comput. Math. 3 (2003), 33107.

[Z] A. Zygmund, Trigonometric Series, Vol. 1, Cambridge Univ. Press, Cambridge, 1977.

Department of Mathematics

Moscow State University

119992 Moscow, Russia

E-mail: konyagin@ok.ru
Department of Mathematics University of South Carolina Columbia, SC 29208, U.S.A. E-mail: temlyak@math.sc.edu 